\title{
17 Soziale Kompetenz in Notfall- und Krisensituationen
}

\author{
Arnd T. May
}

\subsection{Einleitung}

Soziale Kompetenz wird meist als selbstverständliche, intuitiv vorhandene Eigenschaft neben der Beherrschung der fachlichen Kenntnisse vorausgesetzt. Moralische Intuitionen sind zwar meist hilfreich, können aber im Einzelfall nicht eindeutig sein. Soziale Kompetenz im professionellen Alltag erfordert eine Kenntnisvermittlung und eine Praxis samt Reflexion des eigenen Verhaltens. Die Tätigkeit der an der Krankenversorgung beteiligten Personen ist durch Handeln und Aktion geprägt, nicht durch Zuschauen und Kontemplation. In der zeitkritischen Situation der Notfallversorgung steht für viele Handelnde das aktive Moment deutlich im Vordergrund. Dabei wird der Heil- und Hilfsauftrag zum Wohle des Patienten ausgeübt. Selbst in der Situation der lebensrettenden Sofortmaßnahmen darf ärztliches Handeln kein blinder Aktionismus sein oder Handeln um des Handelns willen, sondern muss den Patienten im Blick behalten und die Sinnhaftigkeit der Versorgung, was oft als medizinische oder ärztliche Indikation beschrieben wird.

Die Mitwirkung an der Versorgung in Notfall- und Krisensituationen ist von Einsatzbereitschaft, Verantwortungsbereitschaft und Entscheidungsnotwendigkeiten geprägt. Dies kann bei unterschiedlichen Wertvorstellungen und Überzeugungen der Beteiligten zu Konflikten führen. Neben klaren Entscheidungsbefugnissen ist soziale Kompetenz integraler Faktor für das Gelingen in einer arbeitsteiligen Versorgung. Die Interaktion im Team und mit zeitweise in die Behandlung und Versorgung eingebundenen Personen ist für das gegenseitige Verständnis elementar. 
Verwirklichung der eigenen Ziele bei gleichzeitiger Wahrung der sozialen Akzeptanz des Verhaltens (Kanning 2002: 155).

Für die besondere Situation der Versorgung von Patienten in Notfall- und Krisensituationen und die Kommunikation mit Angehörigen, Vertrauenspersonen oder weiteren am Geschehen beteiligten Personen hilft Wissen über Meinungsbildung und Kommunikation und die Reflexion über psychische Bedürfnisse von Menschen. Sowohl für den Patienten als auch für andere in der Unfallsituation beteiligten Personen ist das traumatische Ereignis ein punktuell einschneidendes Ereignis, welches vorher nicht planbar war und nun mehr oder weniger in der konkreten Situation erlebt wird. Sobald der Rettungsdienst die Behandlung übernommen hat, werden der Takt der Versorgung und zur Behandlung nötige Maßnahmen dem Patienten überwiegend mitgeteilt und nicht wertneutral zur Auswahl präsentiert. Je lebensbedrohlicher die Verletzung ist, umso automatisierter läuft die Versorgung ab. Patienten verlieren in dieser Situation nicht ihr Selbstbestimmungsrecht, sind aber zur vollumfänglichen Wahrnehmung oft nicht in der Lage. Damit der Patient mitwirkt und keine Gegenwehr zu den Maßnahmen leistet, sind Informationen und Begleitung erforderlich. Diese zwischenmenschliche Begleitung erfordert Einfühlungsvermögen und Verständnis für diese existentielle Ausnahmesituation, die der Patient überwiegend das erste Mal in seinem Leben erfährt, wohingegen für Profis im Gesundheitswesen diese Situationen zum rettungsdienstlichen und intensivmedizinischen Alltag gehören.

Das medizinethische Ideal der Einwilligung nach Aufklärung wird häufig situationsbedingt nicht realisierbar sein. Dennoch bleibt es der inzwischen in $\mathbb{5} 630$ BGB normierte Auftrag, dass der Patient „über sämtliche für die Einwilligung wesentlichen Umstände“ aufgeklärt werden muss. Damit der Patient eine wohlüberlegte Entscheidung über die Einwilligung treffen kann, müssen ihm die Informationen rechtzeitig gegeben werden ( $\$$ 6zoe Abs. $2 \mathrm{BCB}$ ). Bei traumatischen Verletzungsmustern wird der weitere Verlauf detailliert kaum abschätzbar sein. Somit können Informationen zu den zu erwartenden Folgen und Risiken der Maßnahme nur situationsbedingt eingeschränkt gegeben werden.

Der Kontakt mit lebensbedrohlich verletzten Menschen und diese Wahrnehmung von existentiellen weil prognostisch unsicheren Situationen können zu einer Abstumpfung gegenüber normalen Gefühlen in diesen Situationen führen. Entscheidend für den persönlichen Umgang mit diesen Alltagssituationen sind Hilfemöglichkeiten, welche am Schluss dieses Beitrags vorgestellt werden.

\subsection{Patienten in Not}

Notfallpatienten sind „Personen, die sich infolge Verletzung, Krankheit oder sonstiger Umstände entweder in Lebensgefahr befinden oder bei denen schwere gesundheitliche Schäden zu befürchten sind, wenn sie nicht unverzüglich medizinische Hilfe erhalten “ ( $\$ 2$ Abs. 2 Satz 3 des Gesetzes über den Rettungsdienst sowie die Notfallrettung und den Krankentransport durch Unternehmer (Rettungsgesetz NRW RettC NRW) vom 24. November 1992). Daraus ergibt sich eine eindeutige Behandlungsund Versorgungspriorität. Personen, die nicht detailliert die Unterschiede der Hilfesysteme im Gesundheitswesen kennen, werden in ihrer Not schnell und bevorzugt die 112 wählen und den Notarzt anfordern, wenn sie „eine für sie unlösbare Situation 
sehen“ und das Dilemma für sich mit dem Rettungsmittel lösen, welches am schnellsten an den Einsatzort kommen wird (Engelbrecht 2015). Die Erwartung von schneller und umfassender Hilfe motiviert zum Notruf und abgesehen von bewussten Fehlalarmierungen befinden sich insbesondere Angehörige in einer für sie unlösbaren Situation, die sie möglichst kurz allein erleben möchten und daher zügige Hilfe anfordern, die auch ihnen Beistand bringt und sie aus der Situation herausholt, für die Versorgung und Begleitung des Patienten allein verantwortlich zu sein. Zusätzlich zu dieser individualethischen Perspektive lohnt die Bewertung der Behandlungspriorität im Einzelfall. Dazu schätzte Dick 2000, dass 30-40\% der Einsätze des Rettungsdienstes eindeutig der vertragsärztlichen Versorgung zuzuordnen sind und damit unter den Sicherstellungsauftrag der Kassenärztlichen Vereinigung fallen (Dick 200o: 491). Die kostenrelevanten Aspekte der Organisation des Rettungsdienstes können hier nicht weiter betrachtet werden. Die Perspektive soll weiter individualethisch auf den Patienten, die Angehörigen und die versorgenden Fachleute gerichtet bleiben.

Patienten befinden sich nach einem traumatischen Ereignis in einer Ausnahmesituation. Ängste können für Patienten entstehen durch:

- Hilflosigkeit, Überforderung, Schmerzen, Kontrollverlust, verändertes Selbsterleben

- Angst vor seelenloser Versorgung als Objekt der Maximaltherapie

- Sorge um Arbeitsplatz, Zukunft, Angehörige

- Scham

In einer Situation der Hilflosigkeit und Überforderung ist für Patienten und auch Angehörige eine klare Kommunikation hilfreich.

Die eingespielte Routine der Versorgung kann als Stressor wirken. Umso bedeutsamer ist die umsichtige, aber klare Kommunikation. Dazu gehören:

- Zuständigkeiten klären und nicht mit mehreren Personen auf den Patienten einreden

- Vorstellen der eigenen Person, Beachtung der Anredeform des Patienten

- klare, verständliche Botschaften, keine „schonenden Umschreibungen“

- Kommunikationsbarrieren ausschließen (z.B. Schwerhörigkeit)

- einfache Sätze mit möglichst einer Botschaft oder Frage („einfache, leichte Sprache“) ohne komplexe Satzkonstruktionen

- Vorbereiten des Patienten auf bevorstehende Maßnahmen

Bei einwilligungsunfähigen oder bewusstseinsgetrübten Patienten ist die Kommunikation erschwert und wird die Beobachtung von leiblichen Ausdrucksformen in den Vordergrund rücken. Bei bewusstlosen Patienten ist die Tendenz zu beobachten, im Beisein des Patienten über diesen zu sprechen.

Für einen wertschätzenden Umgang mit Patienten muss die Kommunikation im Beisein des Patienten über ihn und mit ihm in der Form stattfinden, als wäre er bei Bewusstsein. 
Solange keine gesicherten Informationen über die kognitiven Leistungen des Patienten vorliegen, sollte die Kommunikation normal als Monolog ablaufen und mindestens die Information über Maßnahmen beinhalten. Damit wird standardmäßig eine Information mitgeteilt. Diese Vorgehensweise schützt vor bitteren Überraschungen, wenn vermeintlich Bewusstlose Wahrnehmungen auch während der Bewusstlosigkeit zu berichten wissen.

\title{
17.3 Besondere Patientengruppen
}

Die Vielfalt der Patienten im Gesundheitswesen ist ein Spiegel unserer Gesellschaft und es treffen regelhaft unterschiedliche Wertvorstellungen aufeinander. Die wertschätzende Begegnung ist ein wertvolles Gut und integraler Bestandteil der neutralen Versorgung im Gesundheitswesen. In der Musterberufsordnung für Ärzte steht in $\mathbb{} 7$ Nummer die Verpflichtung, „Patientinnen und Patienten gebührende Aufmerksamkeit entgegen zu bringen“. Eine patientenorientierte Behandlung und Versorgung wird den Patientenwillen beachten und das Patientenwohl im Blick behalten.

Einen Katalog besonderer Patientengruppen zu erstellen bleibt angesichts mannigfaltiger Abgrenzungsschwierigkeiten eine Herausforderung und wird den besonderen Interessen der beteiligten Patienten nicht gerecht. Es gilt die Sensibilität für Patienteninteressen zu schärfen und einen professionellen Umgang mit persönlich herausfordernden Situationen zu trainieren. Dabei sind die Herausforderungen nicht generalisierbar. Aufmerksamkeit fordernde Einflussgrößen können durch den Patienten, seine Angehörigen oder Vertrauenspersonen oder die Einsatzsituation vorliegen. Hilfreich wird die offene Kommunikation sein, mit der im Behandlungsteam die aktuelle Irritation oder persönliche Einschränkung ausgesprochen wird. Für die gewohnte Behandlung störend können Zuschauer sein, welche gaffen, den Einsatz behindern und evtl. dauerhaft fotografieren oder filmen. Diese Verhaltensweisen sind geeignet, dem Patienten zu schaden. Dies mag schon durch den verzögerten Zugang zur Einsatzstelle geschehen oder das Binden von Aufmerksamkeit. Eine deutliche Kommentierung kann helfen und letztlich können die Ordnungskräfte einbezogen werden. Der Appell zum Perspektivwechsel kann autoritativ verstärkt werden.

Laut $\mathbb{2} 2$ der Ausbildungs- und Prüfungsverordnung für Notfallsanitäterinnen und Notfallsanitäter (NotSan-APrV) soll der Unterricht „die Entwicklung der zur Ausübung des Berufs erforderlichen Personal-, Sozial- und Selbstkompetenz“ fördern. In der Ausbildung sind 120 Stunden für Kommunikation und Interaktion mit sowie Beratung von hilfesuchenden und hilfebedürftigen Menschen unter Berücksichtigung des jeweiligen Alters sowie soziologischer und psychologischer Aspekte vorgesehen.

\author{
Notfallsanitäter erhalten in ihrer Ausbildung Informationen zu folgenden \\ besonderen Personengruppen: \\ - sterbende Patienten sowie ihre Angehörigen, \\ - Kinder, \\ - Jugendliche, ältere Menschen, \\ - pflegebedürftige Menschen, \\ - gesellschaftliche Randgruppen, \\ - übergewichtige Menschen,
}




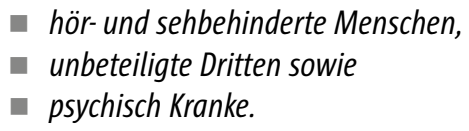

In der rettungsdienstlichen und intensivmedizinischen Literatur tradiert sich die Vignette des über 8o Jahre alten Vaters, der einen Kindernotfall bei der Rettungsleitstelle meldet, und das Notfallteam dann auf die über 50 Jahre alte Tochter trifft, die in häuslicher Gemeinschaft mit den Eltern lebt. Zweifelsfrei sind die Verwandtschaftsverhältnisse zutreffend, aber ohne Altersklärung haben die Rettungsdienstmitarbeiter eine andere Altersspanne ihrer zukünftigen Patientin erwartet. Dies lässt sich nur durch Nachfragen des Leitstellendisponenten klären, wobei im konkreten Beispiel die Schwere der Verletzung ausschlaggebend sein sollte und nicht das Alter. Gleichwohl werden Einsätze mit Kindern aus emotionaler Betroffenheit oft mit einer noch höheren Dringlichkeit bearbeitet.

Für eine kultursensible Gesundheitsversorgung und interkulturelle Kompetenz ist die kritische Auseinandersetzung mit der eigenen kulturellen Identität und deren Einfluss auf die eigenen Bewertungen zentral (Peters et al. 2014: 69). Durch das Bewusstmachen eigener Werte und Überzeugungen wird das Fremde erkennbar. Die Etikettierung eines Patienten mit dem Krankheitsbild „Morbus Bosporus“ ist ebenso diskriminierend wie die Begrenzung der Zahl der Besucher. Ziel der Versorgung in der Akutsituation ist eine patientenorientierte Behandlung. Dabei liegt der Fokus auf den individuellen Wertvorstellungen, Bedürfnissen und Nöten des Einzelnen. Eine Zuordnung von Menschen zu Wertgemeinschaften oder Kulturen mag eine erste Annäherung erleichtern, trägt aber nicht zu einem wertschätzenden Umgang bei, wenn die Annahmen nicht verifiziert und überprüft werden. So muss auch ein Zeuge Jehovas zu seiner aktuellen in der jetzigen Situation vorliegenden Meinung zu Bluttransfusionen nach der entsprechenden Information und Aufklärung befragt werden. Es wäre ein falsch verstandener Umgang mit Überzeugungen, Werten und Informationen, wenn die Information der Glaubenszugehörigkeit zu den Zeugen Jehovas automatisch als Ablehnung einer Bluttransfusion interpretiert würde und dies nicht besprochen würde. Eine solche wertende Annahme ist mit dem Prinzip der Einwilligung oder Ablehnung nach Information und Aufklärung nicht vereinbar. Es würden Vorurteile und unüberprüfte Annahmen unzulässige Geltung erlangen, was mit einer empathischen unbeeinflussten Annährung an den unbekannten und fremden Menschen unvereinbar ist. Vorurteile kommen nicht nur bei Fremden vor, denn der hochbetagte Mensch muss nicht automatisch schwerhörig sein, wenngleich mit höherem Alter die Häufigkeit des eingeschränkten Hörvermögens zunimmt.

Durch die Aufzählung von relevanten ,besonderen“ Patientengruppen mit beachtenswerten Interessen oder beachtenswerten Dingen werden sozialer Status, Geschlecht, Staatsbürgerschaft, Dauer des Aufenthalts in Deutschland nicht als relevante und entscheidungsleitende Faktoren anerkannt. Die Hilfsverpflichtung für notleidende Patienten ist unbedingt und kann als Ausdruck der Humanität unserer Gesellschaft gesehen werden. 


\subsection{Kommunikation im Team}

Die bereits dargestellte klare Kommunikation hilft, Missverständnissen vorzubeugen. Auch wird in Situationen von Zeitnot manche Äußerung sprichwörtlich aufgenommen, selbst wenn die gleiche Aussage in anderen Momenten augenzwinkernd kommentiert wird. Wenn der Notarzt dem Assistenzpersonal mitteilt, dass er erst einmal einen „Zugang“ zum Patienten bekommen möchte, dann bedeutet dies keine Handlungsaufforderung, die Vorbereitungen zum Legen eines peripher venösen $\mathrm{Zu}$ gangs (Venenverweilkanüle) zu beginnen und den Stauschlauch anzureichen.

In Phasen der Zeitnot sollte Kommunikation keine weitere Störgröße darstellen, was aber zweifellos der Fall wäre, wenn der Empfänger erst die Exegese der Botschaft des Senders betreiben müsste. Die Aufmerksamkeit des Kommunikationspartners sollte von der Patientenversorgung nicht auf die Interpretation von Kommunikationsinhalten gelenkt werden, um eine mögliche Ablenkung zu vermeiden. Eine komplizierte Kommunikation ist Quelle für Fehler und Missverständnisse.

Kommunikationsstörungen können entstehen durch:

- Dialekt, Sprechgeschwindigkeit, Tonart,

- Angst, Unsicherheit, Vorurteile,

- Begrifflichkeiten,

- Informationsüberflutung und

- unübersichtliche Darstellung komplexer Vorgänge.

Eine Kommunikation mit einfachen Satzkonstruktionen hilft zur Schaffung von Klarheit. Dies muss nicht gleich zum Befehlston abgleiten aber klar strukturierte Aussagen helfen beiden Kommunikationspartnern. Fehler durch Kommunikationsstörungen können verhindert werden, wenn strukturierte Formen des Austausches Verwendung finden. Damit kann ein vertrautes Miteinander geschaffen werden, welches in unübersichtlichen Situationen der Reiz- und Informationsüberflutung der aktuellen Situation vertraute Sicherheit im Miteinander gibt. Bei der Formulierung von Arbeitsaufträgen untereinander muss schon bei der Formulierung darauf geachtet werden, dass sie verstanden und umgesetzt werden können. Komplexe Vorgänge müssen überschaubar unterteilt werden. Nach der Erledigung von Aufträgen hilft eine Rückmeldung zur Planung der weiteren Maßnahmen.

Gelungene Kommunikation kann zu einer Entlastung in herausfordernden Situationen werden. Dies beinhaltet auch die Achtsamkeit der Wirkung der eigenen Kommunikation auf andere Personen.

\subsection{Hilfsmöglichkeiten}

Der erste Weg zur Unterstützung sollte zu den Kolleginnen und Kollegen sein, da diese in der Regel schnell verfügbar sind. Deren Zeitbudget für die Besprechung akuter Schwierigkeiten wird wegen der eigenen Einbindung in die Patientenversorgung begrenzt sein. Doch für eine erste Rückmeldung samt Wahrnehmungsabgleich und erstem Hinweis auf Lösungsoptionen sollte auch im Arbeitsalltag Zeit sein. Bei einer kollegialen Beratung findet ein offener Austausch innerhalb des Stationsteams oder 
der beteiligten Arbeitseinheit statt. Konkrete Probleme und Praxisfälle werden durch die Rückmeldungen, Wahrnehmungen und Bewertungen der anderen Cruppenmitglieder systematisch reflektiert, um Lösungsoptionen für den Berufsalltag zu entwickeln. Diese als Intervision bezeichnete Möglichkeit des Austausches findet ohne externe Anleitung statt.

Zur Konfliktlösung hat sich das Instrument der Klinischen Ethikberatung bewährt, welches zunehmend im Gesundheitswesen Verbreitung findet. Ethik-Fallberatungen dienen der Unterstützung in schwierigen Entscheidungs- bzw. Behandlungssituationen. Ausgangspunkt für eine Ethik-Fallberatung ist eine unterschiedliche Einschätzung zur weiteren Versorgung oder auch zum Patientenwillen. Beratungen können von allen an der Entscheidung bzw. Behandlung Beteiligten beantragt werden (z.B. Mitarbeitende aus den verschiedenen Berufsgruppen, Patienten und Bewohner, deren Angehörige und Stellvertreter). In einer moderierten, strukturierten EthikFallberatung wird die Klärung des Konflikts angestrebt. Damit dient Ethikberatung der Information, Orientierung und Beratung der verschiedenen an der Versorgung beteiligten bzw. davon betroffenen Personen (z.B. Mitarbeitende und Leitung der Einrichtung, Patienten/Bewohner, deren Angehörige und Stellvertreter im Sinne des Bevollmächtigten oder gesetzlichen Betreuers). Ethikberatung trägt mit einem multiprofessionellen Team von Ethikberatern dazu bei, Probleme und Konflikte möglichst einvernehmlich zu lösen und die erarbeitete Lösung praktisch umzusetzen. Ethische Anteile an Entscheidungsprozessen können sichtbar gemacht werden und Entscheidungen an moralisch akzeptablen Kriterien ausgerichtet werden (d.h. eine „gute“ Entscheidung in einem „guten“ Entscheidungsprozess zu treffen). Ethik-Fallberatungen finden überwiegend zu aktuellen noch nicht entschiedenen Situationen als prospektive Besprechungen statt, können aber auch retrospektiv Entscheidungssituationen reflektieren (May 2012).

Eine Supervision kann je nach Konflikt als Einzelgespräch oder mit dem Team stattfinden. In einer Einzelsupervision steht persönliches Verhalten im Vordergrund, wozu insbesondere die individuellen Wertvorstellungen, Einstellungen, Orientierungen Erfahrungen, Gedanken und Gefühle angesprochen und auf die Konfliktsituation bezogen werden. Bei einer Cruppenvision wird die Zusammenarbeit im Team bzw. in der Organisation mit den beteiligten Personen des Kollektivs besprochen werden. Auf unterschiedlichen Ebenen des professionellen Miteinanders kann ein Beitrag zu deren Miteinander und deren Beziehung geleistet werden. Der Supervisor ist regelmäßig eine externe qualifizierte Person, welche in die Dynamik der Arbeitssituation nicht eingebunden ist.

Für die Qualifizierung, Fort- und Weiterbildung von Stationsteams sind Maßnahmen sinnvoll, die jene Themenfelder auffrischen, die in der ausschnittweise dargestellten Ausbildungs- und Prüfungsverordnung für Notfallsanitäterinnen und Notfallsanitäter beschrieben sind. Ziel der Schulungen ist der professionelle Umgang mit anderen Wertvorstellungen, die sonst aus Unkenntnis zur Verstörung und Ablehnung führen könnten. Weltoffenheit und Toleranz macht sich durch soziale Kompetenz im Gesundheitswesen bemerkbar. 


\section{Literatur}

Dick WF (2000) „Tun oder Nichtstun“ - das ist hier die Frage. Notfall und Rettungsmedizin 3: 491

Engelbrecht K (2015) Rettungsdienst, lebensrettende Sofortmaßnahmen und Aspekte der apparativen Intensivmedizin. In: May A, Kreß H, Verrel T, Wagner T (Hg.) Patientenverfügungen. Handbuch für Berater, Ärzte und Betreuer. Springer, Heidelberg, S. 189-207

Kanning U (2002) Soziale Kompetenz - Definition, Strukturen und Prozesse. Zeitschrift für Psychologie 210: 154163

May A (2012) Professionalisierung und Standardisierung der Ethikberatung. In: Frewer A, Bruns F, May A (Hg.) Ethikberatung in der Medizin. Springer, Heidelberg, S. 65-75

May A, Mann R (2005) Soziale Kompetenz im Notfall. 2. Aufl. LIT, Münster

Peters T, Grützmann T, Bruchhausen W, Coors M, Jacobs F, Kaelin L, Knipper M, Kressing F, Neitzke G (2014) Grundsätze zum Umgang mit Interkulturalität in Einrichtungen des Gesundheitswesens. Ethik Med 26: 65-75

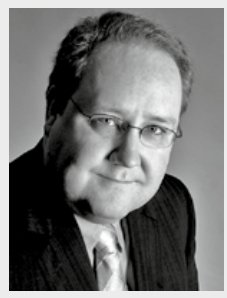

\section{Dr. phil. Arnd T. May}

ethikzentrum.de - Zentrum für Angewandte Ethik Recklinghausen

Studium der Philosophie mit dem Schwerpunkt Angewandte Ethik, Medizinethik. Promotion zu Patientenverfügungen. Zertifizierter Trainer für Ethikberatung im Gesundheitswesen (AEM).

Aufbau Klinischer Ethikberatung an zwei Universitätsklinika in Deutschland und weiteren Einrichtungen des Gesundheitswesens. 